\title{
On the set of complex points of a 2-sphere
}

\author{
NIKOLAY SHCHERBINA
}

\begin{abstract}
Let $G$ be a strictly pseudoconvex domain in $\mathbb{C}^{2}$ with $C^{\infty}$-smooth boundary $\partial G$. Let $S$ be a 2-dimensional sphere embedded into $\partial G$. Denote by $\mathcal{E}$ the set of all complex points on $S$. We study how the structure of the set $\mathcal{E}$ depends on the smoothness of $S$.
\end{abstract}

Mathematics Subject Classification (2000): $32 \mathrm{~T} 15$ (primary): 32V40, 53D10 (secondary).

\section{Introduction}

Let $M$ be a 2-dimensional $C^{1}$-smooth manifold in $\mathbb{C}^{2}$. A point $p$ on $M$ is called a complex point, if the tangent plane $T_{p} M$ to $M$ at $p$ is a complex line. Denote by $\mathcal{E}$ the set of all complex points on $M$. If $M$ is smooth enough and in a general position, then the set $\mathcal{E}$ consists of isolated points. In this case the topology of $M$ can be described in terms of the local behaviour of $M$ near the points of $\mathcal{E}$ (see [11]). The structure of the set $M$ near the points in $\mathcal{E}$ plays a key role in different questions of complex analysis (see, for example, [2,3,9,10,12,15]. In this paper we study the structure of the set $\mathcal{E}$ in the case, when $M$ is a 2-dimensional sphere, denoted by $S$ in what follows, embedded into the boundary $\partial G$ of a $C^{\infty}$-smooth strictly pseudoconvex domain $G$ in $\mathbb{C}^{2}$ (this case is important for applications, as was shown in [5] and [6]). It is easy to see that, due to strict pseudoconvexity of $G$, the set $\mathcal{E}$ has no interior points in $S$. Our goal here is to give a more explicit description of the set $\mathcal{E}$ depending on the smoothness of $S$. Recall, that a manifold is said to be of class $C^{2-}$ if it can be represented locally as the graph of a function that belongs to the class $\operatorname{Lip}^{1, \alpha}$ for each positive $\alpha<1$. Our main result can now be formulated as follows.

Theorem 1.1. Let $G$ be a strictly pseudoconvex domain in $\mathbb{C}^{2}$ with $C^{\infty}$-smooth boundary $\partial G$. Let $S$ be a 2-dimensional sphere embedded into $\partial G$. Then, depending on the smoothness of $S$, the following holds:

1) If $S$ is of class $C^{2}$, then the set $\mathcal{E}$ of complex points of $S$ is contained in a $C^{1}$ smooth nonclosed curve $\gamma \subset S$.

Received January 11, 2008; accepted July 9, 2008. 
2) There exists a 2-dimensional sphere $S \subset \partial G$ of class $C^{2-}$ such that the set $\mathcal{E}$ contains a Jordan curve of positive 2-dimensional measure.

ACKNOWLEDGEMENTS. Part of this work was done while the author was a visitor at the Max Planck Institute of Mathematics (Bonn) and at the Scuola Normale Superiore (Pisa). It is my pleasure to thank these institutions for their hospitality and excellent working conditions. I also express my gratitude to N. G. Kružilin for valuable comments.

\section{Proof of the first part of the theorem}

We start with an argument which goes back to Bishop [2] (see also [9]). Namely, if $p$ is a point of $\mathcal{E}$, then after a polynomial change of coordinates that moves $p$ to the origin we can locally represent $S$ as the disc

$$
D=\left\{(z, f(z)) \in \mathbb{C}^{2}: z \in \Delta\right\}
$$

with $\triangle$ being a small disc centered at the origin and $f$ being a complex valued $C^{2}$-smooth function. Moreover, in view of strict pseudoconvexity of $G$, after this change of coordinates the function $f$ will have the special form

$$
f(z)=\frac{1}{2}|z|^{2}-\beta \operatorname{Re} z^{2}+o\left(|z|^{2}\right), \quad \beta \geq 0
$$

near zero. Recall, that zero is called an elliptic point if $0 \leq \beta<\frac{1}{2}$, a hyperbolic point if $\beta>\frac{1}{2}$ and a parabolic point if $\beta=\frac{1}{2}$. Elliptic and hyperbolic points are always isolated in $\mathcal{E}$. In the case of a parabolic point we can use the real coordinates $z=x+i y$ and represent $f$ as $f(z)=y^{2}+o\left(|z|^{2}\right)$. Hence $\partial_{\bar{z}} f(z)=i y+o(|z|)$ and then, by the implicit function theorem, we obtain that $\sigma=\left\{z \in \Delta: \operatorname{Im} \partial_{\bar{z}} f(z)=0\right\}$ is a $C^{1}$-smooth curve and locally $\mathcal{E}=$ $\left\{(z, f(z)): z \in \sigma\right.$ and $\left.\operatorname{Re} \partial_{\bar{z}} f(z)=0\right\}$. Therefore, locally the set $\mathcal{E}$ is a closed subset of a $C^{1}$-smooth curve.

Since the set $\mathcal{E}$ is compact, the only obstruction for $\mathcal{E}$ to be a subset of a nonclosed $C^{1}$-smooth curve $\gamma \subset S$ is that there is a closed $C^{1}$-smooth curve $\Gamma \subset \mathcal{E}$.

Assume, to get a contradiction, that such a closed $C^{1}$-smooth curve $\Gamma \subset \mathcal{E}$ exists. Consider a complex tangential $C^{\infty}$-smooth closed curve $\Gamma^{\prime}$ in $\partial G$ (complex tangential here means that for each point $p \in \Gamma^{\prime}$ the tangent line $T_{p} \Gamma^{\prime}$ to $\Gamma^{\prime}$ at $p$ is contained in the complex tangent plane $T_{p}^{\mathbb{C}}(\partial G)$ to $\partial G$ at $p$ ) close enough to $\Gamma$ in the $C^{1}$-metric. Then, using a partition of unity along the curve $\Gamma^{\prime}$, we can construct a small $C^{1}$-perturbation $S^{\prime}$ of $S$ in $\partial G$ such that $\Gamma^{\prime} \subset S^{\prime}$ and each point in $\Gamma^{\prime}$ is a complex point on $S^{\prime}$. Moreover, $S^{\prime}$ can be made $C^{\infty}$-smooth in a neighbourhood of $\Gamma^{\prime}$. For each point $p \in \Gamma^{\prime}$, consider the unit vector $\vec{u}(p)$ tangent to $\Gamma^{\prime}$, the vector 
$i \vec{u}(p) \in T_{p}^{\mathbb{C}}(\partial G)$ and the unit vector $\vec{n}(p) \in T_{p}(\partial G)$ orthogonal to $T_{p}^{\mathbb{C}}(\partial G)$ and such that the vectors $(\vec{u}(p), i \vec{u}(p), \vec{n}(p))$ define the positive orientation of $\partial G$ at the point $p$. Let $O(p)$ be the rotation of $T_{p}(\partial G)$ around the direction $\vec{u}(p)$ that transforms the vector $i \vec{u}(p)$ into the vector $\vec{n}(p)$. Using the tubular neighbourhood theorem (see, for example, [8, Theorem 1.4]) we can change $S^{\prime}$ in a neighbourhood of $\Gamma^{\prime}$ to get a new 2-sphere, $S^{\prime \prime} \subset \partial G, C^{\infty}$-smooth near $\Gamma^{\prime}$ such that $\Gamma^{\prime} \subset S^{\prime \prime}$ and $\vec{n}(p) \in T_{p}\left(S^{\prime \prime}\right)$ for each point $p \in \Gamma^{\prime}$. It is easy to see that $S^{\prime \prime}$ is totally real near $\Gamma^{\prime}$. Then we can perturb $S^{\prime \prime}$ slightly outside a small neighbourhood of $\Gamma^{\prime}$ to get a $C^{\infty}$-smooth 2-sphere $\tilde{S} \subset \partial G$ in general position. To finish the proof of the first part of our theorem we use an argument of Gromov (see [7, page 342]). Namely, by the result of Bedford-Klingenberg [3] and Kružilin [10], there is a smooth 3-ball $\mathcal{B}$ which is the disjoint union of holomorphic discs $\left\{D_{\alpha}\right\}$, such that $\partial \mathcal{B}=\tilde{S}$. By Chirka's theorem [4] we know that discs $D_{\alpha}$ are $C^{\infty}$-smooth to the boundary $\partial D_{\alpha}$ near the totally real part of $\tilde{S}$ (i.e. outside of finitely many complex points of $\tilde{S}$ ) and, moreover, the boundary $\partial D_{\alpha}$ of each disc $D_{\alpha}$ is $C^{\infty}$-smooth at this part of $\tilde{S}$ and transversal there to the distribution $\left\{T_{p}^{\mathbb{C}}(\partial G)\right\}$ of complex tangencies to $\partial G$. Consider a disc $D_{\alpha_{0}}$ from the family $\left\{D_{\alpha}\right\}$ such that its boundary $\partial D_{\alpha_{0}}$ "touches" the curve $\Gamma^{\prime}$ "for the first time" and let $p$ be a point of $\partial D_{\alpha_{0}} \cap \Gamma^{\prime}$. More precisely, let $D_{\alpha_{0}} \subset \mathcal{B}$ be a holomorphic disc with the property that $\partial D_{\alpha_{0}} \cap \Gamma^{\prime} \neq \varnothing$ and such that for some connected component of the set $\mathcal{B} \backslash D_{\alpha_{0}}$, each holomorphic disc $D_{\alpha}$, which is contained in this component, satisfies $\partial D_{\alpha} \cap \Gamma^{\prime}=\emptyset$. Now we can see that, on the one hand, since the curves $\partial D_{\alpha_{0}}$ and $\Gamma^{\prime}$ are tangent to each other at the point $p$, and since the curve $\Gamma^{\prime}$ was chosen to be complex tangential, the curve $\partial D_{\alpha_{0}}$ is complex tangential at $p$. On the other hand, since the point $p$ is contained in the totally real part of $\tilde{S}$, the curve $\partial D_{\alpha_{0}}$ has to be transversal to $T_{p}^{\mathbb{C}}(\partial G)$. This gives the desired contradiction and completes the proof of the first part of the theorem.

Remark 2.1. In the special case when the boundary of the domain $G$ is diffeomorphic to a 3-dimensional sphere, the fact that the closed complex tangential curve $\Gamma \subset S \subset \partial G$ mentioned above does not exist can also be deduced from the $[1$, Theorem 1].

\section{Proof of the second part of the theorem}

We prove the second part of the theorem in several steps. First, we construct a special arc $E \subset \mathbb{R}_{x, y}^{2}$ of positive 2-dimensional measure. Then we define a function $G$ on $E$ such that $G \in C^{2-}(E)$ with the functions $G_{x}^{\prime}(x, y)=y$ and $G_{y}^{\prime}(x, y)=0$ chosen to be the first derivatives of $G$ on $E$. Next, following an idea of $\mathrm{H}$. Whitney (see [14]), we construct a nonconstant function $H \in C^{2-}(E)$ with $H_{x}^{\prime}(x, y)=0$ and $H_{y}^{\prime}(x, y)=0$. Using $G$ and $H$ we define a function $F \in C^{2-}(E)$ with $F_{x}^{\prime}(x, y)=y$ and $F_{y}^{\prime}(x, y)=0$ which is zero at both endpoints of $E$. Then, using $E$, we construct a Jordan curve $\tilde{E} \subset \mathbb{R}_{x, y}^{2}$ of positive 2-dimensional measure and, using $F$, we define a function $\tilde{F}$ on $\tilde{E}$ of class $C^{2-}(\tilde{E})$ with derivatives 
$\tilde{F}_{x}^{\prime}(x, y)=y$ and $\tilde{F}_{y}^{\prime}(x, y)=0$. Next, applying Whitney's extension theorem to the function $\tilde{F}$, we contruct a 2 -sphere $S^{2} \subset \mathbb{R}_{x, y, z}^{3}$ of class $C^{2-}$ which contains a Jordan curve of positive 2-dimensional measure such that at each point of this curve the tangent plane to $S^{2}$ coincides with the corresponding plane of the standard contact distribution in $\mathbb{R}_{x, y, z}^{3}$. Finally, using the Darboux theorem, we embed this sphere into the boundary $b G$ of the given strictly pseudoconvex domain $G$.

\subsection{Construction of the $\operatorname{arc} E$}

First, we define for each $\alpha \in(0,1)$ an auxiliary set

$$
\begin{aligned}
\mathbb{E}^{\alpha}= & \left(\left[0, \frac{1-\alpha}{2}\right] \cup\left[\frac{1+\alpha}{2}, 1\right]\right) \times\left(\left[0, \frac{1-\alpha}{2}\right] \cup\left[\frac{1+\alpha}{2}, 1\right]\right) \\
& \cup(\{0\} \times[0,1]) \cup\left([0,1] \times\left\{\frac{1+\alpha}{2}\right\}\right) \cup(\{1\} \times[0,1]) .
\end{aligned}
$$

We denote $A=(0,0), B=(1,0), Q_{0}=\left[0, \frac{1-\alpha}{2}\right] \times\left[0, \frac{1-\alpha}{2}\right], Q_{1}=\left[0, \frac{1-\alpha}{2}\right] \times$ $\left[\frac{1+\alpha}{2}, 1\right], Q_{2}=\left[\frac{1+\alpha}{2}, 1\right] \times\left[\frac{1+\alpha}{2}, 1\right]$, and $Q_{3}=\left[\frac{1+\alpha}{2}, 1\right] \times\left[0, \frac{1-\alpha}{2}\right]$. Further, we denote $A_{0}=A=(0,0), B_{0}=\left(0, \frac{1-\alpha}{2}\right), A_{1}=\left(0, \frac{1+\alpha}{2}\right), B_{1}=\left(\frac{1-\alpha}{2}, \frac{1+\alpha}{2}\right), A_{2}=$ $\left(\frac{1+\alpha}{2}, \frac{1+\alpha}{2}\right), B_{2}=\left(1, \frac{1+\alpha}{2}\right), A_{3}=\left(1, \frac{1-\alpha}{2}\right)$, and $B_{3}=B=(1,0)$ (see the set $\mathbb{E}^{\alpha}$ in Figure 3.1).

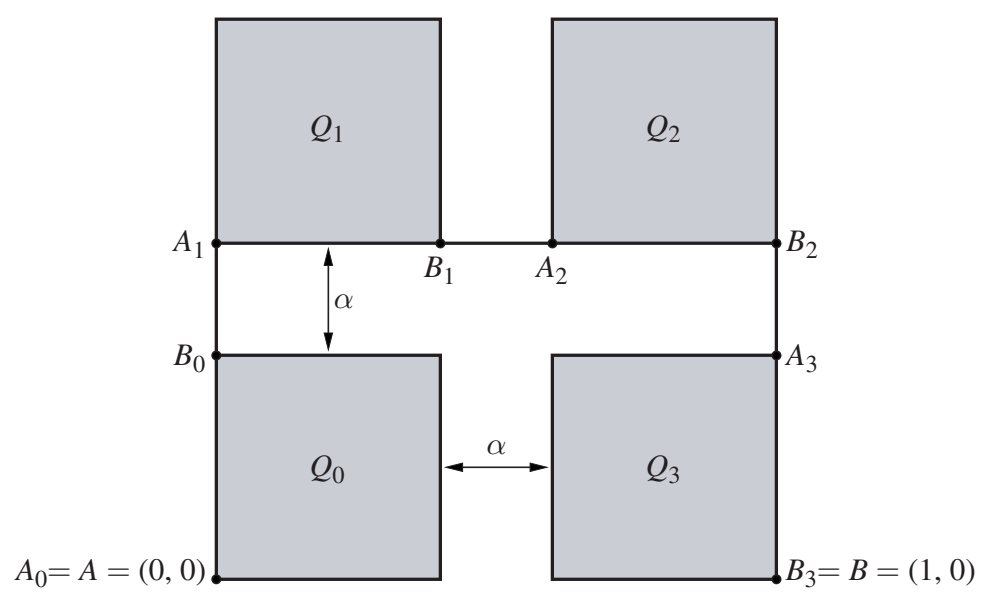

Figure 3.1. The set $\mathbb{E}^{\alpha}$.

To define the set $E$ we consider the sequence $\alpha_{n}=\frac{1}{(n+1)^{2}}, n=1,2, \ldots$ We construct the set $E$ as the intersection of a decreasing sequence of compact sets $E_{n}$ which will be defined inductively. We set $E_{1}=\mathbb{E}^{\alpha_{1}}$. To define the set $E_{2}$ we consider the image $\tilde{\mathbb{E}}^{\alpha_{2}}$ of the set $\mathbb{E}^{\alpha_{2}}$ under the homothety with coefficient $\frac{1-\alpha_{1}}{2}$. 
Then for each $i=0,1,2,3$ we consider the set $\mathbb{E}_{i}$ obtained from the set $\tilde{\mathbb{E}}^{\alpha_{2}}$ by an orthogonal transformation (if necessary) and translation in such a way that the image of the points $A$ and $B$ will coincide with the points $A_{i}$ and $B_{i}$, respectively. It is easy to see that we need an orthogonal transformation only for $i=0,3$. The set $E_{2}$ is obtained from the set $E_{1}$ by replacing for each $i=0,1,2,3$ the square $Q_{i}$ by the corresponding set $\mathbb{E}_{i}$. For each $j=0,1,2,3$ we denote by $Q_{i j}, A_{i j}$ and $B_{i j}$ the images of the square $Q_{j}$ and the points $A_{j}, B_{j}$ in the corresponding set $\mathbb{E}_{i}$, respectively (the set $E_{2}$ is shown in Figure 3.2).

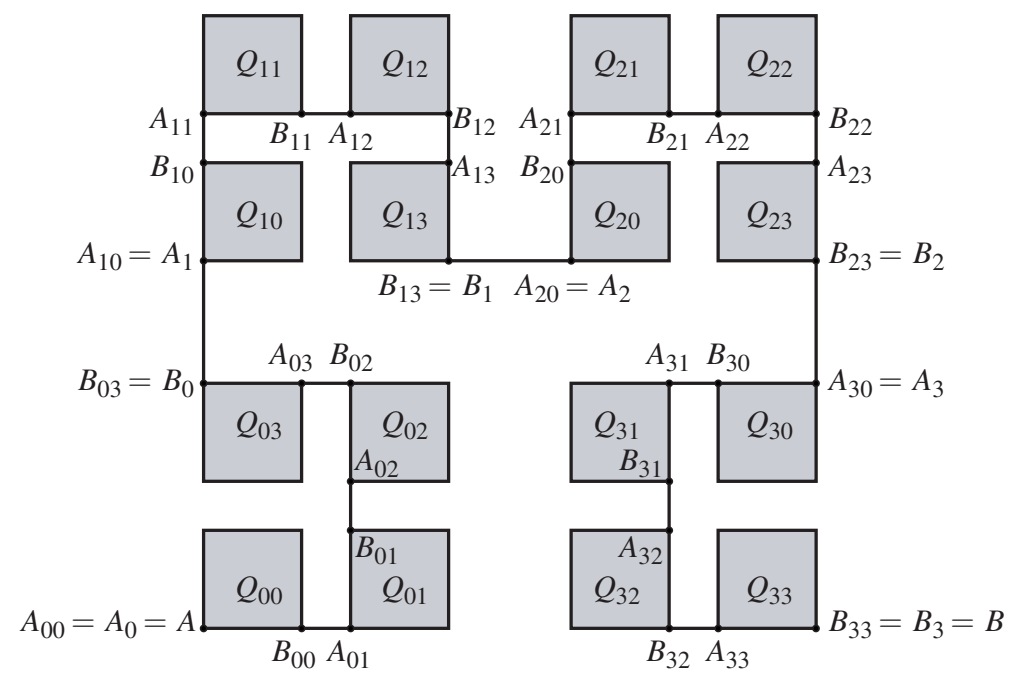

Figure 3.2. The set $E_{2}$.

To describe the inductive step of our construction we assume that the set $E_{n}$ is already constructed and define the set $E_{n+1}$. Consider the image $\tilde{\mathbb{E}}^{\alpha_{n+1}}$ of the set $\mathbb{E}^{\alpha_{n+1}}$ under the homothety with coefficient $\prod_{i=1}^{n}\left(\frac{1-\alpha_{i}}{2}\right)$. Then for each multiindex $\left(i_{1}, i_{2}, \ldots, i_{n}\right), i_{j}=0,1,2,3, j=1,2, \ldots, n$, consider the set $\mathbb{E}_{i_{1} \ldots i_{n}}$ obtained from the set $\tilde{\mathbb{E}}^{\alpha_{n+1}}$ by an orthogonal transformation (if necessary) and translation in such a way that the image of the points $A$ and $B$ will coincide with the points $A_{i_{1} \ldots i_{n}}$ and $B_{i_{1} \ldots i_{n}}$, respectively. The set $E_{n+1}$ is obtained from the set $E_{n}$ by replacing each square $Q_{i_{1} \ldots i_{n}}$ by the corresponding set $\mathbb{E}_{i_{1} \ldots i_{n}}$. For each $i_{j}=0,1,2,3, j=$ $1,2, \ldots, n+1$, we denote by $Q_{i_{1} \ldots i_{n+1}}, A_{i_{1} \ldots i_{n+1}}$ and $B_{i_{1} \ldots i_{n+1}}$ the images of the square $Q_{i_{n+1}}$ and the points $A_{i_{n+1}}, B_{i_{n+1}}$ in the corresponding set $\mathbb{E}_{i_{1} \ldots i_{n}}$, respectively. Note, that for each multiindex $\left(i_{1}, \ldots, i_{n}\right)$ one has $A_{i_{1} \ldots i_{n} 0}=A_{i_{1} \ldots i_{n}}$ and $B_{i_{1} \ldots i_{n} 3}=B_{i_{1} \ldots i_{n}}$.

Since $\left\{E_{n}\right\}$ is a decreasing sequence of compact sets, $E=\bigcap_{n=1}^{\infty} E_{n}$ is a nonempty compact subset of $\mathbb{R}_{x, y}^{2}$. It is easy to see that it is an arc (see the set $E$ in Figure 3.3). 


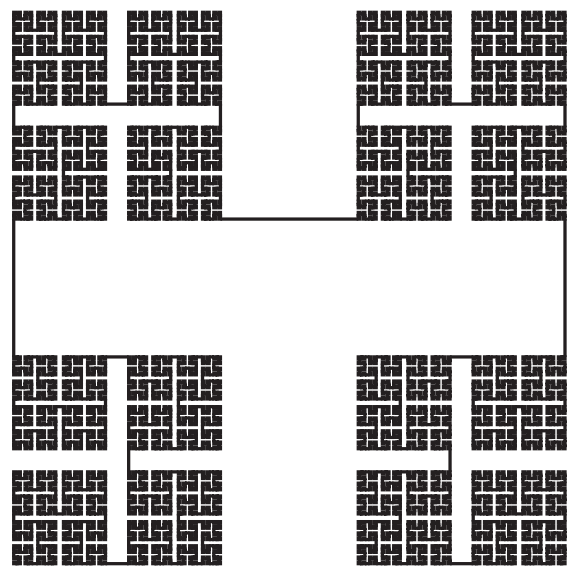

Figure 3.3. The set $E$.

To estimate the area of the set $E$ we observe that

$$
\begin{aligned}
\text { Area }\left(E_{n}\right)=\left(1-\alpha_{n}\right)^{2} \text { Area }\left(E_{n-1}\right) & =\prod_{k=1}^{n}\left(1-\alpha_{k}\right)^{2}=\prod_{k=1}^{n}\left(1-\frac{1}{(k+1)^{2}}\right)^{2} \\
& =\left(\frac{1}{2}\left(1+\frac{1}{n+1}\right)\right)^{2}>\frac{1}{4}
\end{aligned}
$$

for every $n=1,2, \ldots$ Hence, the set $E$ has a positive 2-dimensional measure (and, moreover, Area $\left.(E)=\frac{1}{4}\right)$.

\subsection{Definition and properties of the function $G$}

For each $n=1,2, \ldots$ let $\Omega_{n}$ be the connected component of the set $(0,1) \times(-1,1) \backslash$ $E_{n}$ containing the square $(0,1) \times(-1,0)$ and let $J_{n}=\partial \Omega_{n} \cap([0,1] \times[0,1])$. On each curve $J_{n}$ we define a function $G_{n}$ in the following way. For a point $p \in J_{n}$ we denote by $J_{n}^{p}$ a part of $J_{n}$ with initial point $A$ and endpoint $p$ and then we set $G_{n}(p)=\int_{J_{n}^{p}} y d x$.

We will need the following estimate on the function $G_{n}$.

Lemma 3.1. Let $Q_{i_{1} i_{2} \ldots i_{m}}$ be a square obtained after $m \geq 6$ steps of our construction above. Then for each natural number $n>m$ and all points $p, q \in J_{n} \cap Q_{i_{1} i_{2} \ldots i_{m}}$ one has

$$
\left|G_{n}(q)-G_{n}(p)-\int_{[p, q]} y d x\right|<\operatorname{Area}\left(Q_{i_{1} i_{2} \ldots i_{m}}\right)=\frac{1}{2^{2 m+2}}\left(\frac{m+2}{m+1}\right)^{2} .
$$

Proof. We proceed by induction on $m$ having the number $n$ fixed and decreasing the number $m$. For each pair of points $r, s \in J_{n}$ we denote by $J_{n}^{r, s}$ the part of the curve 
$J_{n}$ with the endpoints $r$ and $s$. For $m=n-1$ we observe from the definition of the function $G_{n}$ and Green's theorem that $G_{n}(q)-G_{n}(p)-\int_{[p, q]} y d x$ represents the sum (with signes) of the areas of domains bounded by the $\operatorname{arc}_{n}^{p, q}$ and the segment $[p, q]$ (see Figure 3.4).

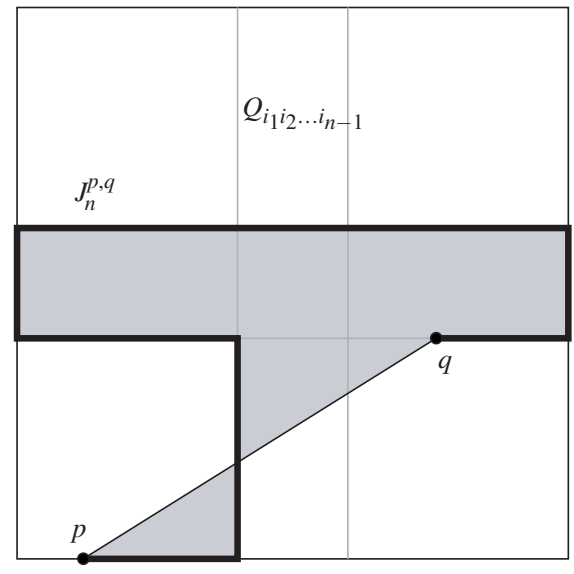

Figure 3.4. The curve $J_{n}^{p, q}$ in the square $Q_{i_{1} i_{2} \ldots i_{n-1}}$.

Since these domains are contained in the square $Q_{i_{1} i_{2} \ldots i_{n-1}}$, we conclude that the inequality (3.1) holds true in this case. The same argument cannot be applied in the case of general $m$ due to the fact that the corresponding domains will not be disjoint anymore and, therefore, their areas will be counted with multiplicities. That is why in the case of an arbitrary $m$ we need to improve the argument above and to use the inductive procedure.

Assume that the inequality (3.1) holds true for all squares $Q_{i_{1} i_{2} \ldots i_{m}}$ and all points $p, q \in J_{n} \cap Q_{i_{1} i_{2} \ldots i_{m}}$ with $m \geq m_{0}+1$ ( $n$ being fixed). Consider a square $Q_{i_{1} i_{2} \ldots i_{m_{0}}}$ and points $p, q \in J_{n} \cap Q_{i_{1} i_{2} \ldots i_{m_{0}}}$. Observe first that the segment [ $\left.p, q\right]$ can intersect at most three of the squares $Q_{i_{1} i_{2} \ldots i_{m_{0}} i_{m_{0}+1}}, i_{m_{0}+1}=0,1,2,3$. We assume in what follows that it intersects exactly three of them (in the other cases the argument is easier, since some terms in our estimates will disappear), namely the squares $Q_{i_{1} i_{2} \ldots i_{m_{0}} 0}, Q_{i_{1} i_{2} \ldots i_{m_{0}} 1}$ and $Q_{i_{1} i_{2} \ldots i_{m_{0}} 2}$ (the cases of another three squares can be treated similarly). Consider an orientation of the segment $[p, q]$ from the point $p$ to the point $q$ and for each $i=0,1,2$ denote by $p_{i}$ and $p_{i}^{\prime}$ the first and the last, respectively, (according to the orientation of $[p, q]$ ) points of the set $[p, q] \cap$ $J_{n}^{p, q} \cap Q_{i_{1} i_{2} \ldots i_{m_{0}} i}$. Note, that $p_{0}=p$ and $p_{2}^{\prime}=q$. For each $i=0,1,2$ we have by our inductive hypotesis that

$\left|G_{n}\left(p_{i}^{\prime}\right)-G_{n}\left(p_{i}\right)-\int_{\left[p_{i}, p_{i}^{\prime}\right]} y d x\right|<\operatorname{Area}\left(Q_{i_{1} i_{2} \ldots i_{m_{0}} i}\right)<\frac{1}{4} \operatorname{Area}\left(Q_{i_{1} i_{2} \ldots i_{m_{0}}}\right)$.

Since the segment $\left[p_{0}^{\prime}, p_{1}\right]$ intersects the curve $J_{n}$ only at its endpoints, we conclude from the Green's theorem that $G_{n}\left(p_{1}\right)-G_{n}\left(p_{0}^{\prime}\right)-\int_{\left[p_{0}^{\prime}, p_{1}\right]} y d x$ represents the 
area of the domain $\Sigma_{1}$ bounded by the curve $J_{n}^{p_{0}^{\prime}, p_{1}}$ and the segment $\left[p_{0}^{\prime}, p_{1}\right]$ (see Figure 3.5).

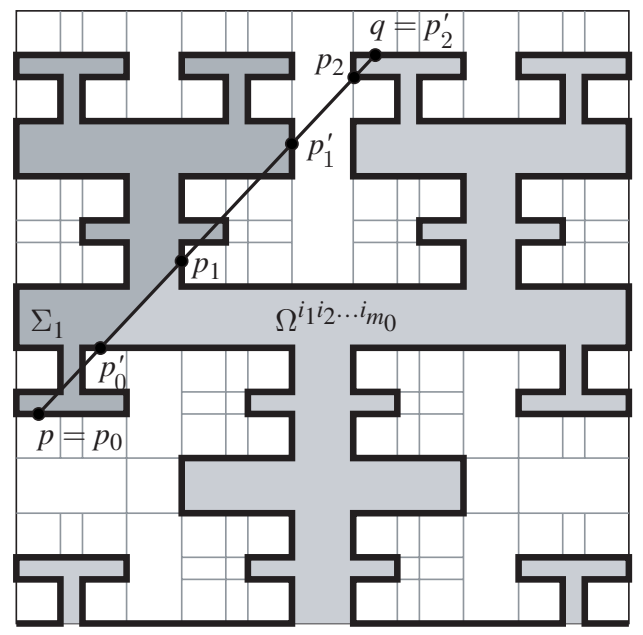

Figure 3.5. The sets $\Sigma_{1}$ and $\Omega_{n}^{i_{1} i_{2} \ldots i_{m_{0}}}$ in the square $\Omega_{i_{1} i_{2} \ldots i_{m_{0}}}$.

Since the domain $\Sigma_{1}$ is contained in the set $\Omega_{n}^{i_{1} i_{2} \ldots i_{m_{0}}}=\Omega_{n} \cap Q_{i_{1} i_{2} \ldots i_{m_{0}}}$, and since $\Omega_{n} \cap Q_{i_{1} i_{2} \ldots i_{m_{0}}} \subset Q_{i_{1} i_{2} \ldots i_{m_{0}}} \backslash E_{n}$, it follows that

$$
\begin{aligned}
0 & <G_{n}\left(p_{1}\right)-G_{n}\left(p_{0}^{\prime}\right)-\int_{\left[p_{0}^{\prime}, p_{1}\right]} y d x=\operatorname{Area}\left(\Sigma_{1}\right)<\operatorname{Area}\left(\Omega_{n} \cap Q_{i_{1} i_{2} \ldots i_{m_{0}}}\right) \\
& <\operatorname{Area}\left(Q_{i_{1} i_{2} \ldots i_{m_{0}}}\right)-\operatorname{Area}\left(E_{n} \cap Q_{i_{1}, i_{2} \ldots i_{m_{0}}}\right) \\
& =\operatorname{Area}\left(Q_{i_{1} i_{2} \ldots i_{m_{0}}}\right)\left(1-\left(1-\alpha_{m_{0}+1}\right)^{2} \ldots\left(1-\alpha_{n}\right)^{2}\right) \\
& =\operatorname{Area}\left(Q_{i_{1} i_{2} \ldots i_{m_{0}}}\right)\left(1-\left(1-\frac{1}{\left(m_{0}+2\right)^{2}}\right)^{2} \ldots\left(1-\frac{1}{(n+1)^{2}}\right)^{2}\right) \\
& =\operatorname{Area}\left(Q_{i_{1} i_{2} \ldots i_{m_{0}}}\right)\left(1-\left(\frac{\left(m_{0}+1\right)(n+2)}{\left(m_{0}+2\right)(n+1)}\right)^{2}\right) \\
& <\operatorname{Area}\left(Q_{i_{1} i_{2} \ldots i_{m_{0}}}\right)\left(1-\left(\frac{m_{0}+1}{m_{0}+2}\right)^{2}\right)<\frac{2}{m_{0}+2} \text { Area }\left(Q_{i_{1} i_{2} \ldots i_{m_{0}}}\right) .
\end{aligned}
$$

Similarly, $G_{n}\left(p_{2}\right)-G_{n}\left(p_{1}^{\prime}\right)-\int_{\left[p_{1}^{\prime}, p_{2}\right]} y d x$ represents the area with the negative sign (due to the orientation of its boundary) of the domain $\Sigma_{2}$ bounded by the curve $J_{n}^{p_{1}^{\prime}, p_{2}}$ and the segment $\left[p_{1}^{\prime}, p_{2}\right]$. Let $\Pi$ be the smallest rectangle in $Q_{i_{1} i_{2} \ldots i_{m_{0}}}$ that coutains the squares $Q_{i_{1} i_{2} \ldots i_{m_{0}}} 12, Q_{i_{1} i_{2} \ldots i_{m_{0}}} 13, Q_{i_{1} i_{2} \ldots i_{m_{0}} 20}$ and $Q_{i_{1} i_{2} \ldots i_{m_{0}}} 21$ (see Figure 3.6). 


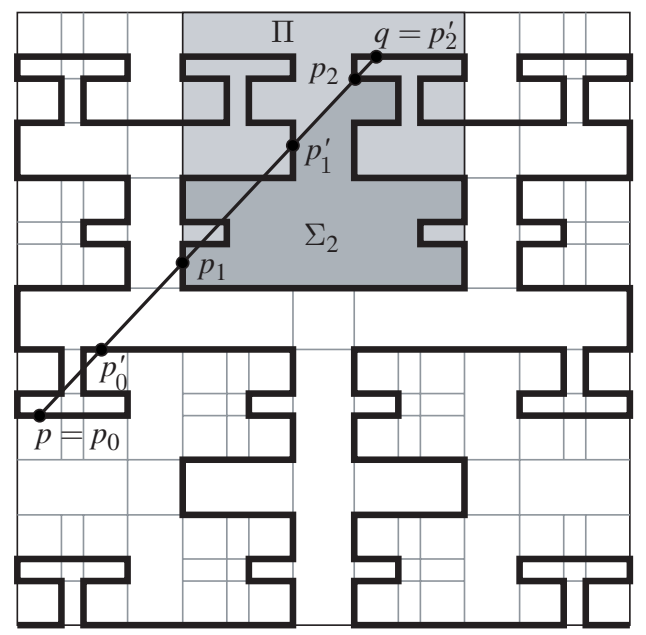

Figure 3.6. The sets $\Sigma_{2}$ and $\Pi$ in the square $\Omega_{i_{1} i_{2} \ldots i_{m_{0}}}$.

Since the domain $\Sigma_{2}$ is a subdomain of $\Pi$, and since we have an obvious estimate Area $(\Pi)<\frac{1}{4}$ Area $\left(Q_{i_{1} i_{2} \ldots i_{m_{0}}}\right)$, we conclude that

$$
\begin{aligned}
0>G_{n}\left(p_{2}\right)-G_{n}\left(p_{1}^{\prime}\right)-\int_{\left[p_{1}^{\prime}, p_{2}\right]} y d x & =-\operatorname{Area}\left(\Sigma_{2}\right)>-\operatorname{Area}(\Pi) \\
& >-\frac{1}{4} \operatorname{Area}\left(Q_{i_{1} i_{2} \ldots i_{m_{0}}}\right) .
\end{aligned}
$$

Taking into account different signs of the terms in the inequalities (3.3) and (3.4) we have for $m_{0} \geq 6$ the following estimate

$$
\begin{aligned}
& \left|\left(G_{n}\left(p_{1}\right)-G_{n}\left(p_{0}^{\prime}\right)-\int_{\left[p_{0}^{\prime}, p_{1}\right]} y d x\right)+\left(G_{n}\left(p_{2}\right)-G_{n}\left(p_{1}^{\prime}\right)-\int_{\left[p_{1}^{\prime}, p_{2}\right]} y d x\right)\right| \\
& <\frac{1}{4} \operatorname{Area}\left(Q_{i_{1} i_{2} \ldots i_{m_{0}}}\right) .
\end{aligned}
$$

Finally, the inequalites (3.2) and (3.5) imply that

$$
\begin{aligned}
& \left|G_{n}(q)-G_{n}(p)-\int_{[p, q]} y d x\right|<\sum_{i=0}^{2} \mid\left(G_{n}\left(p_{i}^{\prime}\right)-G_{n}\left(p_{i}\right)-\int_{\left[p_{i}, p_{i}^{\prime}\right]} y d x \mid\right. \\
& +\mid\left(G_{n}\left(p_{1}\right)-G_{n}\left(p_{0}^{\prime}\right)-\int_{\left[p_{0}^{\prime}, p_{1}\right]} y d x\right)+\left(G_{n}\left(p_{2}\right)-G_{n}\left(p_{1}^{\prime}\right)-\int_{\left[p_{1}^{\prime}, p_{2}\right]} y d x \mid\right. \\
& <\frac{3}{4} \text { Area }\left(Q_{i_{1} i_{2} \ldots i_{m_{0}}}\right)+\frac{1}{4} \text { Area }\left(Q_{i_{1} i_{2} \ldots i_{m_{0}}}\right)=\text { Area }\left(Q_{i_{1} i_{2} \ldots i_{m_{0}}}\right) .
\end{aligned}
$$

This completes the proof of the lemma. 
Now we define the function $G$ on the $\operatorname{arc} E$. Let $p$ be a point of $E$. Consider a sequence of points $p_{n} \in J_{n}, n=1,2, \ldots$, such that $p_{n} \rightarrow p$, as $n \rightarrow \infty$ and set $G(p)=\lim _{n \rightarrow \infty} G_{n}\left(p_{n}\right)$.

To prove that the function $G$ is well defined we consider the Cantor set $\mathbb{Q} \stackrel{\text { def }}{=}$ $\bigcap_{n=1}^{\infty} \bigcup_{\left(i_{1}, i_{2}, \ldots, i_{n}\right)} Q_{i_{1} i_{2} \ldots i_{n}}$ and observe that since the set $E \backslash \mathbb{Q}$ is constituted by horizontal and vertical segments, and in view of the definition of the functions $G_{n}$, it is enough to prove that the sequence $G_{n}\left(p_{n}\right)$ converges for $p_{n} \rightarrow p$ with $p, p_{n} \in \mathbb{Q}, n=1,2, \ldots$ Let $p_{n_{1}}$ and $p_{n_{2}}, n_{1}>n_{2}$, be two points of our sequence $p_{n} \in J_{n} \cap \mathbb{Q}, n=1,2, \ldots$, and let $Q_{i_{1} i_{2} \ldots i_{m}}$ be the smallest of the described above squares that contains both of these points. Observe that the vertex $A_{i_{1} i_{2} \ldots i_{m}}$ of the square $Q_{i_{1} i_{2} \ldots i_{m}}$ is contained in both curves $J_{n_{1}}$ and $J_{n_{2}}$. It follows then from Green's theorem that $G_{n_{1}}\left(A_{i_{1} i_{2} \ldots i_{m}}\right)-G_{n_{2}}\left(A_{i_{1} i_{2} \ldots i_{m}}\right)$ represents the area of the set

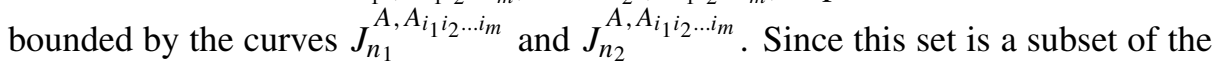
set $\Omega_{n_{1}} \backslash \bar{\Omega}_{n_{2}}$, we conclude that

$$
\left|G_{n_{1}}\left(A_{i_{1} i_{2} \ldots i_{m}}\right)-G_{n_{2}}\left(A_{i_{1} i_{2} \ldots i_{m}}\right)\right|<\operatorname{Area}\left(\Omega_{n_{1}} \backslash \bar{\Omega}_{n_{2}}\right) .
$$

For each $j=1,2$ we have (by the lemma):

$$
\left|G_{n_{j}}\left(p_{n_{j}}\right)-G_{n_{j}}\left(A_{i_{1} i_{2} \ldots i_{m}}\right)-\int_{\left[A_{\left.i_{1} i_{2} \ldots i_{m}, p_{n_{j}}\right]}\right.} y d x\right|<\operatorname{Area}\left(Q_{i_{1} i_{2} \ldots i_{m}}\right) .
$$

If we denote by $l_{m}$ the length of a side of $Q_{i_{1} i_{2} \ldots i_{m}}$ (it depends only on $m$ ), then the last inequality gives us

$$
\begin{aligned}
& \sum_{j=1}^{2}\left|G_{n_{j}}\left(p_{n_{j}}\right)-G_{n_{j}}\left(A_{i_{1} i_{2} \ldots i_{m}}\right)\right|<\sum_{j=1}^{2}\left|\int_{\left[A_{i_{1} i_{2} \ldots i_{m}}, p_{n_{j}}\right]} y d x\right|+2 \operatorname{Area}\left(Q_{i_{1} i_{2} \ldots i_{m}}\right) \\
& <2 l_{m}+2 \operatorname{Area}\left(Q_{i_{1} i_{2} \ldots i_{m}}\right)<3 l_{m}=3 \prod_{i=1}^{m}\left(\frac{1-\alpha_{i}}{2}\right)=\frac{3}{2^{m+1}} \cdot \frac{m+2}{m+1}
\end{aligned}
$$

It follows now from (3.6) and (3.7) that

$$
\begin{aligned}
& \left|G_{n_{1}}\left(p_{n_{1}}\right)-G_{n_{2}}\left(p_{n_{2}}\right)\right| \leq \sum_{j=1}^{2}\left|G_{n_{j}}\left(p_{n_{j}}\right)-G_{n_{j}}\left(A_{i_{1} i_{2} \ldots i_{m}}\right)\right| \\
& +\left|G_{n_{1}}\left(A_{i_{1} i_{2} \ldots i_{m}}\right)-G_{n_{2}}\left(A_{i_{1} i_{2} \ldots i_{m}}\right)\right|<\frac{3}{2^{m+1}} \cdot \frac{m+2}{m+1}+\operatorname{Area}\left(\Omega_{n_{1}} \backslash \bar{\Omega}_{n_{2}}\right) .
\end{aligned}
$$

Since for points $p, p_{n_{1}}, p_{n_{2}} \in \mathbb{Q}$ the number $m$ will tend to infinity as $p_{n_{1}}, p_{n_{2}} \rightarrow$ $p$, and since Area $\left(\Omega_{n_{1}} \backslash \bar{\Omega}_{n_{2}}\right) \rightarrow 0$ as $n_{1}, n_{2} \rightarrow \infty$, we conclude that the limit of the sequence $G_{n}\left(p_{n}\right)$ as $n \rightarrow \infty$ exists and, therefore, the function $G$ is well defined. 
Next, we prove that $G \in C^{2-}(E)$ with $G_{x}^{\prime}(x, y)=y$ and $G_{y}^{\prime}(x, y)=0$ chosen to be the first derivatives of $G$ at each point $(x, y) \in E$. To do this rigorously we recall the definition of a function belonging to the class $C^{2-}(E)$ (this definition is due to H. Whitney. Further details can be found, for example, in [13]).

Definition 3.2. Let $E$ be a compact subset of $\mathbb{R}_{x, y}^{2}$ and let $f$ be a function defined on $E$. We say that $f$ belongs to the class $C^{2-}(E)$ if there exist bounded functions $f_{x}^{\prime}$ and $f_{y}^{\prime}$ defined on $E$ with the property that for each $\varepsilon>0$ there is a constant $M$ such that

$$
\begin{aligned}
& \left|f(x+\Delta x, y+\Delta y)-f(x, y)-f_{x}^{\prime}(x, y) \Delta x-f_{y}^{\prime}(x, y) \Delta y\right| \\
& \leq M(|\Delta x|+|\Delta y|)^{2-\varepsilon}
\end{aligned}
$$

for all $(x, y),(x+\Delta x, y+\Delta y) \in E$.

To prove that $G \in C^{2-}(E)$ we consider two points $p, p+\Delta p \in E$. Since the function $G$ obviously is smooth on each of the segments $\left[B_{i_{1} \ldots i_{n}}, A_{i_{1} \ldots\left(i_{n}+1\right)}\right], i_{n}=$ $0,1,2$, and satisfies condition (3.8) with $G_{x}^{\prime}(x, y)=y$ and $G_{y}^{\prime}(x, y)=0$ there, the general case, when $p, p+\Delta p \in E$, can be easily reduced to the case when $p$ and $p+\triangle p$ are contained in the Cantor set $\mathbb{Q}$. That is why we assume in what follows that $p, p+\Delta p \in \mathbb{Q}$. Consider as above a number $m$ such that $p, p+\Delta p$ belong to a square $Q_{i_{1} \ldots i_{m}}$ for some indices $\left(i_{1}, \ldots, i_{m}\right)$, but not to a smaller square $Q_{i_{1}, \ldots i_{m} i_{m+1}}, i_{m+1}=0,1,2,3$. Since $p$ and $p+\Delta p$ belong to different squares $Q_{i_{1} \ldots i_{m} i_{m+1}}$ and $Q_{i_{1} \ldots i_{m} i_{m+1}^{\prime}}$, it follows that the distance between these points is not less than the minimal distance between $Q_{i_{1} \ldots i_{m} i_{m+1}}$ and $Q_{i_{1} \ldots i_{m} i_{m+1}^{\prime}}$, that is,

$$
\begin{aligned}
|\Delta p| \geq \alpha_{m+1}\left(\frac{1-\alpha_{1}}{2}\right) \ldots\left(\frac{1-\alpha_{m}}{2}\right) & =\frac{1}{(m+2)^{2}} \cdot \frac{1}{2^{m}} \prod_{k=1}^{m}\left(1-\frac{1}{(k+1)^{2}}\right) \\
& =\frac{1}{2^{m+1}} \cdot \frac{1}{(m+1)(m+2)}
\end{aligned}
$$

Now we estimate the left hand side of the condition (3.8) for our function $G$

$$
\begin{aligned}
\mathcal{L}_{G}(p, p+\Delta p) & \stackrel{\text { def }}{=} G(p+\Delta p)-G(p)-G_{x}^{\prime}(p) \Delta x-G_{y}^{\prime}(p) \Delta y \\
& =G(p+\Delta p)-G(p)-y \Delta x,
\end{aligned}
$$

where $p=(x, y)$ and $\Delta p=(\Delta x, \Delta y)$. It is easy to see that

$$
\int_{[p, p+\Delta p]} y d x=y \Delta x+\frac{1}{2} \Delta x \Delta y
$$

hence

$$
\left|\mathcal{L}_{G}(p, p+\Delta p)\right| \leq\left|G(p+\Delta p)-G(p)-\int_{[p, p+\Delta p]} y d x\right|+\frac{1}{2}|\Delta x||\Delta y| .
$$


It follows from the lemma above that

$$
\left|G(p+\Delta p)-G(p)-\int_{[p, p+\Delta p]} y d x\right| \leq \frac{1}{2^{2 m+2}}\left(\frac{m+2}{m+1}\right)^{2} .
$$

Since $p, p+\Delta p \in Q_{i_{1} \ldots i_{m}}$, one can estimate $|\Delta x|$ and $|\Delta y|$ from above by the length of the side of $Q_{i_{1} \ldots i_{m}}$, that is, by $\frac{1}{2^{m+1}}\left(\frac{m+2}{m+1}\right)$. Therefore, we have by (3.10) and (3.11) that

$$
\left|\mathcal{L}_{G}(p, p+\Delta p)\right| \leq \frac{3}{2} \cdot \frac{1}{2^{2 m+2}}\left(\frac{m+2}{m+1}\right)^{2} .
$$

Finally, we conclude from the estimates (3.9) and (3.12) that in order to prove, that $G$ satisfies condition (3.8) we only need to verify that for each $\varepsilon>0$ there is a constant $M$ such that

$$
\frac{3}{2} \cdot \frac{1}{2^{2 m+2}}\left(\frac{m+2}{m+1}\right)^{2} \leq M\left(\frac{1}{2^{m+1}} \cdot \frac{1}{(m+1)(m+2)}\right)^{2-\varepsilon} \text { as } m \rightarrow \infty
$$

which is equivalent to the inequality

$$
\frac{1}{\left(2^{\varepsilon}\right)^{m+1}} \leq M \frac{2}{3}\left(\frac{1}{(m+1)(m+2)}\right)^{2-\varepsilon}\left(\frac{m+1}{m+2}\right)^{2} \text { as } m \rightarrow \infty .
$$

The last inequality is obviously satisfied, since the left hand side tends to zero much faster than the right hand side, as $m \rightarrow \infty$. This proves that the function $G$ belongs to the class $C^{2-}(E)$.

\subsection{Definition and properties of the functions $H$ and $F$}

First, we define the function $H$ on the Cantor set $\mathbb{Q}$. Each point $p$ in this set is uniquely determined as the intersection of the decreasing sequence $Q_{i_{1}} \supset Q_{i_{1} i_{2}} \supset$ $Q_{i_{1} i_{2} i_{3}} \supset \ldots$ of the squares $Q_{i_{1} \ldots i_{n}}$. Then, we define the value of $H$ at the point $p$ as $H(p)=\sum_{n=1}^{\infty} \frac{i_{n}}{4^{n}}$. It is easy to see that for each $i_{n}=0,1,2$ one has $H\left(A_{i_{1} \ldots i_{n-1}\left(i_{n}+1\right)}\right)=\sum_{k=1}^{n} \frac{i_{k}}{4^{k}}+\frac{1}{4^{n}}$ and $H\left(B_{i_{1} \ldots i_{n-1} i_{n}}\right)=\sum_{k=1}^{n} \frac{i_{k}}{4^{k}}+\sum_{k=n+1}^{\infty} \frac{3}{4^{k}}=$ $\sum_{k=1}^{n} \frac{i_{k}}{4^{k}}+\frac{1}{4^{n}}$, therefore, we can extend the function $H$ as a constant to each segment $\left[B_{i_{1} \ldots i_{n-1} i_{n}}, A_{i_{1} \ldots i_{n-1}\left(i_{n}+1\right)}\right], i_{n}=0,1,2$, with the value $\sum_{k=1}^{n} \frac{i_{k}}{4^{k}}+\frac{1}{4^{n}}$ there. This defines the function $H$ on the whole set $E$.

Now we show that $H \in C^{2-}(E)$ with the functions $H_{x}^{\prime}(x, y)=0$ and $H_{y}^{\prime}(x, y)=$ 0 chosen to be the first derivatives of $H$ on $E$. We proceed in the same way as in the case of the function $G$, namely, we consider two points $p, p+\Delta p \in E$. Since, by definition, $H$ is a constant on each of the intervals constituting the set $E \backslash \mathbb{Q}$, we only need to verify that the function $H$ satisfies condition (3.8) for points $p, p+\Delta p \in \mathbb{Q}$. Let, as above, $m$ be a number such that $p, p+\Delta p \in Q_{i_{1} \ldots i_{m}}$, but 
$p, p+\Delta p \notin Q_{i_{1} \ldots i_{m} i_{m+1}}$ for any $i_{m+1}=0,1,2,3$. Then the definition of $H$ gives us that $|H(p+\Delta p)-H(p)| \leq \frac{1}{4^{m}}$. Hence, by estimate (3.9), it is enough to show that for each $\varepsilon>0$ there is $M$ such that

$$
\frac{1}{4^{m}} \leq M\left(\frac{1}{2^{m+1}} \cdot \frac{1}{(m+1)(m+2)}\right)^{2-\varepsilon} \text { as } m \rightarrow \infty,
$$

which is obviously true with the same argument as above for the function $G$.

To define the function $F$ on the set $E$ we first note that by definition of $H$ one has $H(A)=0$ and $H(B)=1$. Then, since by definition of $G$ we have $G(A)=0$, there is a constant $C$ such that for the function $F=G+C H$ one has $F(A)=0$ and $F(B)=0$. Finally, we observe that since $G \in C^{2-}(E)$ with $G_{x}^{\prime}(x, y)=y$ and $G_{y}^{\prime}(x, y)=0$, and since $H \in C^{2-}(E)$ with $H_{x}^{\prime}(x, y)=0$ and $H_{y}^{\prime}(x, y)=0$, it follows that $F \in C^{2-}(E)$ with $F_{x}^{\prime}(x, y)=y$ and $F_{y}^{\prime}(x, y)=0$ at each point $(x, y) \in E$.

\subsection{Construction of the sphere $S \subset \partial G$}

Let $\mathbb{A}$ be the linear transformation of $\mathbb{R}_{x, y}^{2}$ represented by the matrix $\left(\begin{array}{rr}0 & 1 \\ -1 & 0\end{array}\right)$. Consider the sets $E^{1}=E+\vec{e}_{y}, E^{2}=-\mathbb{A} E+\vec{e}_{x}+\vec{e}_{y}, E^{3}=-E+\vec{e}_{x}$ and $E^{4}=\mathbb{A} E$, where $\vec{e}_{x}$ and $\vec{e}_{y}$ are the unit vectors in the coordinate directions $x$ and $y$, respectively, and then define the set $\tilde{E}$ as $\tilde{E}=\bigcup_{i=1}^{4} E^{i}$ (the set $\tilde{E}$ is shown in Figure 3.7).

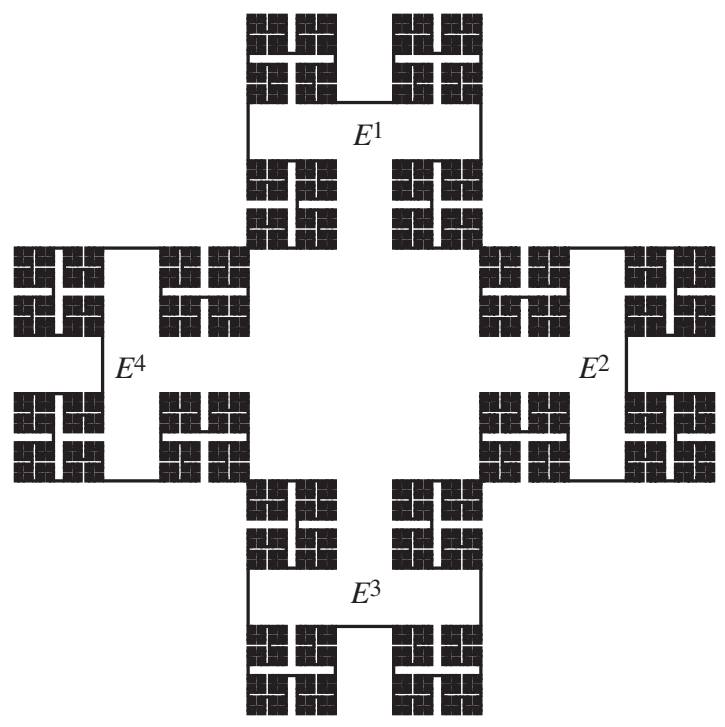

Figure 3.7. The set $\tilde{E}$. 
It is easy to see that $\tilde{E}$ is a Jordan curve of positive 2-dimensional measure in $\mathbb{R}_{x, y}^{2}$. Applying to each of the sets $E^{i}, i=1,2,3,4$, a construction similar to the one that we had above for the function $F$ on the set $E$, we will get functions $F^{i}$ defined on the corresponding sets $E^{i}$ with the properties:

1) $F^{i} \in C^{2-}\left(E^{i}\right)$,

2) $\frac{\partial F^{i}}{\partial x}(x, y)=y$ and $\frac{\partial F^{i}}{\partial y}(x, y)=0$ for each $(x, y) \in E^{i}$,

3) $F^{i}$ has zero values at the endpoints of the arc $E^{i}$.

Hence, we can define a function $\tilde{F}$ on the set $\tilde{E}$ as $\tilde{F}(p)=F^{i}(p)$ for $p \in$ $E^{i}, i=1,2,3,4$, and for this function we will obviously obtain $\tilde{F} \in C^{2-}(\tilde{E})$ with $\frac{\partial \tilde{F}}{\partial x}(x, y)=y$ and $\frac{\partial \tilde{F}}{\partial y}(x, y)=0$ at each point $(x, y) \in \tilde{E}$. Then, by the classical extension theorem of Whitney (see, for example, [13, Theorem 4 on page 177]), there is a function $\widetilde{\widetilde{F}} \in C^{2-}\left(\mathbb{R}_{x, y}^{2}\right)$ such that $\widetilde{\widetilde{F}} \in C^{\infty}\left(\mathbb{R}_{x, y}^{2} \backslash \tilde{E}\right)$ and $\widetilde{\widetilde{F}}(p)=\tilde{F}(p)$ for each $p \in \tilde{E}$. If we restrict the function $\widetilde{\widetilde{F}}$ to a disc $\mathbb{D} \subset \mathbb{R}_{x, y}^{2}$ such that $\tilde{E} \subset \mathbb{D}$ and consider a smooth extension of the graph of this restriction to a 2-dimensional sphere $S^{2}$ embedded into $\mathbb{R}_{x, y, z}^{3}$, then the graph $\Gamma(\tilde{F})$ of $\tilde{F}$ will be a Jordan curve in $S^{2}$ of positive 2-dimensional measure and at each point of this curve the tangent plane to $S^{2}$ will coincide with the corresponding plane of the standard contact distribution $\{d z-y d x=0\}$.

Now let $G$ be a given strictly pseudoconvex domain in $\mathbb{C}^{2}$ with $C^{\infty}$-smooth boundary and let $q$ be a point of $\partial G$. Then, by the theorem of Darboux, there is a neighbourhood $U$ of $q$ in $\partial G$ and a $C^{\infty}$-smooth diffeomorphism $\Phi$ of $U$ onto a neighbourhood $V$ of the origin in $\mathbb{R}_{x, y, z}^{3}$ such that the distribution of complex tangencies $\left\{T_{p}^{\mathbb{C}}(\partial G)\right\}$ will be transformed by $\Phi$ to the standard contact distribution in $\mathbb{R}_{x, y, z}^{3}$. We can assume without loss of generality that $S^{2} \subset V$ (if not, we consider a linear transformation $x \rightarrow c x, y \rightarrow c y, z \rightarrow c^{2} z$ of $\mathbb{R}_{x, y, z}^{3}$ which preserves the standard contact structure and use the image of $S^{2}$ under this transformation with $c>0$ sufficiently small instead of $\left.S^{2}\right)$. Then $S=\Phi^{-1}\left(S^{2}\right)$ will be a 2-dimensional sphere in $\partial G$ of class $C^{2-}$ and the set $\Gamma=\Phi^{-1}(\Gamma(\tilde{F})) \subset S$ will be a Jordan curve of positive 2-dimensional measure such that at each point $p \in \Gamma$ the tangent plane $T_{p} S$ to $S$ is a complex line. This proves the second part of the theorem.

\section{References}

[1] D. Bennequin, Entrelacements et équations de Pfaff, Astérisque 107-108 (1983), 87-161.

[2] E. BISHOP, Differentiable manifolds in complex Euclidean space, Duke Math. J. 32 (1965), $1-21$.

[3] E. BeDford and W. KLINGEnBerG, On the envelope of holomorphy of a 2-sphere in $\mathbb{C}^{2}$, J. Amer. Math. Soc. 4 (1991), 623-643.

[4] E. M. ChIRKA, Regularity of the boundaries of analytic sets, Mat. Sb. 117 (1982), 291336; English transl. in Sb. Math. 45 (1983), 291-335. 
[5] Ya. Eliashberg, Filling by holomorphic discs and its applications, Geometry of lowdimensional manifolds, 2 (Durham, 1989), London Math. Soc. Lecture Note Ser., Vol. 151, Cambridge Univ. Press, Cambridge, 1990, 45-67.

[6] O. G. ERoshKIN, On a topological property of the boundary of an analytic subset of a strictly pseudoconvex domain in $\mathbb{C}^{2}$, Mat. Zametki 49 (1991), 149-151; English transl. in Math. Notes 49 (1991), 546-547.

[7] M. Gromov, Pseudoholomorphic curves in symplectic manifolds, Invent. Math. 82 (1985), 307-347.

[8] M. W. HiRSCH, "Differential Topology", Grad. Texts in Math., Vol. 33, Springer-Verlag, New York, 1976.

[9] B. JÖRICKE, Local polynomial hulls of discs near isolated parabolic points, Indiana Univ. Math. J. 46 (1997), 789-826.

[10] N. G. KRUŽILIN, Two-dimensional spheres on the boundaries of pseudoconvex domains in $\mathbb{C}^{2}$, Izv. Akad. Nauk SSSR Ser. Mat. 55 (1991), 1194-1237; English transl. in Math. USSR Izv. 39 (1992), 1151-1187.

[11] H. F. LAI, Characteristic classes of real manifolds immersed in complex manifolds, Trans. Amer. Math. Soc. 172 (1972), 1-33.

[12] S. NemIROVSKI, Complex analysis and differential topology on complex surfaces, Uspekhi Mat. Nauk 54 (1999), 47-74; English transl. in Russian Math. Surveys 54 (1999), 729-752.

[13] E. SteIN, "Singular Integrals and Differentiability Properties of Functions", Princeton Univ. Press, Princeton, 1970.

[14] H. WHITNEY, A function not constant on a connected set of critical points, Duke Math. J. 1 (1935), 514-517.

[15] J. WIEGERINCK, Local polynomially convex hulls at degenerated CR singularities of surfaces in $\mathbb{C}^{2}$, Indiana Univ. Math. J. 44 (1995), 897-915.

Department of Mathematics

University of Wuppertal

42119 Wuppertal, Germany

shcherbina@math.uni-wuppertal.de 